\title{
Irregular operations on distributions
}

J. MIKUSIŃSKI (Katowice)

§1. Introduction. Several operations on functions are extended to operations on distributions, e.g. addition, multiplication, convolution, Fourier transform ete. Some of them (e. g. addition) are always feasible. Other, not always feasible will be called irregular operations. The aim of this paper is to give a general method of defining irregular operations which are an extension of corresponding operations of functions. We shall base this method upon the sequential theory of distributions which has been exposed in the paper [3]. That paper is concerned with distributions of one variable only, but after slight modifications it is also valid in the case of several variables [4]. Some explanations and necessary notation will be given in $\S 2$.

Several ideas in this paper are the result of common discussions with R. Sikorski.

§ 2. Generalities and notation. Points of the $q$-dimensional space are noted

$$
x=\left(\xi_{1}, \ldots, \xi_{q}\right), \quad a=\left(a_{1}, \ldots, a_{q}\right), \quad k=\left(x_{1}, \ldots, x_{q}\right) \quad \text { ete. }
$$

By $a \leqslant x$ we understand $\alpha_{i} \leqslant \xi_{i}(i=1, \ldots, q)$. Similarly, by $a<x$ we understand $a_{i}<\xi_{i}(i=1, \ldots, q)$. The meaning of $q$-dimensional intervals $a \leqslant x \leqslant b$ and $a<x<b$ is clear.

The functions of class $C^{\infty}$ will be called smooth functions. By the $k$-th derivative of a smooth function $\varphi(x)=\varphi\left(\xi_{1}, \ldots, \xi_{q}\right)$ we understand

$$
\varphi^{(k)}(x)=\frac{\partial^{x_{1}+\ldots+x_{q}}}{\partial \xi_{1}^{\alpha_{1} \ldots \partial \xi_{q}^{q_{q}}}} \varphi\left(\xi_{1}, \ldots, \xi_{q}\right) .
$$

We shall also need the integral

$$
\int_{a}^{x} \varphi(t) d t=\int_{\alpha_{1}}^{\xi_{1}} d \tau_{1} \ldots \int_{a_{q}}^{\xi_{q}} \varphi\left(\tau_{1}, \ldots, \tau_{q}\right) d \tau_{q} .
$$

A sequence of smooth functions $\varphi_{n}(x)$ is said to be fundamental if for every interval $a \leqslant x \leqslant b$ there exist an order $k$ and smooth functions 
$\Phi_{n}(x)$ such that $\Phi_{n}^{(k)}=\varphi_{n}(x)$ and the sequence $\Phi_{n}(x)$ converges uniformly. Two fundamental sequences $\varphi_{n}(x)$ and $\psi_{n}(x)$ are equivalent if the sequence $\varphi_{1}(x), \psi_{1}(x), p_{2}(x), \psi_{2}(x), \ldots$ is fundamental. The only difference in comparison with analogous notions in [3] is that we take here smooth functions instead of continuous functions. The definition of distributions as classes of equivalent fundamental sequenees and other definitions remain the same. The distribution defined by a fundamental sequence $\phi_{n}(x)$ will be denoted by $\left[p_{n}(x)\right]$.

In 0-dimensional case $(q=0)$ the smooth functions are numbers. Almost uniformly convergent sequences and fundamental sequences are convergent sequences of numbers. The distributions are also nothing else but numbers. The 0 -dimensional case is entirely trivial, but the inclusion of it unifies some notions in the general theory.

3. Regular operations. Before introducing irregular operations it seems to be convenient to say a few words upon regular operations. In general, we shall deal with operations which assign to finite systems of smooth functions $\varphi, \psi, \ldots$ a smooth function $R(p, \psi, \ldots)$; these operations will be extended to operations on distributions. An operation $R(p, \psi, \ldots)$ is said to be regular, if for any fundamental sequences $p_{n}, \psi_{n}, \ldots$ the sequence $R\left(p_{n}, \psi_{n}, \ldots\right)$ is also fundamental. The following operations are examples of regular operations:

$1^{0}$ Addition: $\varphi(x)+\psi(x)$;

$2^{\circ}$ Subtraction: $\varphi(x)-\psi(x)$;

$3^{\circ}$ Multiplication by a number $c: o p(x)$;

$4^{0}$ Translation by a vector $c: p(x-c)$;

$5^{0}$ Multiplication by a fixed smooth function $\omega(x): \omega(x) \varphi(x)$;

$6^{\circ}$ Substitution of a fixed smooth function $\omega(x)$ with a non vanishing gradient in the place of a one-dimensional variable: $\varphi(\omega(x))$;

$7^{\circ}$ Direct product: $\varphi(x) \psi(y)$;

$8^{\circ}$ Derivation of an arbitrary order $k: p^{(k)}(x)$;

$9^{\circ}$ Convolution by a smooth function with bounded support $\omega(x)$ : $\omega(x) * \varphi(x)$;

$$
\text { 10 Integral: } \int_{x}^{y} \varphi(t) d t .
$$

Of course, the number of examples could be arbitrarily multiplied. The operations $1^{\circ}, 2^{\circ}, 6^{\circ}$ and $7^{\circ}$ are performed on pairs of functions, the other on a single function. Operations on systems of several functions can be obtained by a superposition of a finite number of regular operations; in this way we always obtain regular operations. In $1^{\circ}-6^{\circ}, 8^{\circ}$ and $9^{\circ}$, the result of the operation and the functions on which it is performed have the same dimensions (the number of variables is the same). In $7^{\circ}$ the dimension of the result is $q+r$, when denoting respectively by $q$ and $r$ the dimensions of $\varphi$ and $\psi$. In $10^{\circ}$, the dimension of the result is $2 q$, when denoting by $q$ the dimension of $\varphi$.

Every regular operation is extended automatically to operations on arbitrary distributions $f, g, \ldots$, on putting

$$
R(f, g, \ldots)=\left[R\left(\varphi_{n}, \psi_{n}, \ldots\right)\right],
$$

where $f=\left[\varphi_{n}\right], g=\left[\psi_{n}\right], \ldots$ This extended operation is always feasible and determined uniquely [2].

4. Irregular operations. The multiplication $\varphi(x) \psi(x)$, when considered as an operation on two functions, is not regular, since the sequence $\varphi_{n}(x) \psi_{n}(x)$ is, in general, not fundamental, although the sequences $\varphi_{n}(x)$ and $\psi_{n}(x)$ are both fundamental. Similarly the substitution $\varphi(\psi(x))$ is not a regular operation on two functions; even if the interior function is a fixed smooth function, but nothing else is supposed on it. For instance, if $\psi(x)$ is a constant function $\psi(x)=c$, the substitution $\varphi(e)$ is not a regular operation.

An operation which is not regular will be called irregular. Such an operation can not be extended as to be feasible on all distributions, but in general it is possible to obtain a weaker extension which is feasible on some distributions. For instance the product can be defined for some pairs of distributions, similarly the substitution of a constant function into a distribution is feasible in some particular cases, as it has been showed by S. Eojasiewiez.

Let us list some of important irregular operations which are concerned with the theory of distributions:

$11^{\circ}$ Multiplication: $\varphi(x) \psi(x)$ :

$12^{\circ}$ Substitution: $\varphi(\psi(x))$;

$13^{\circ}$ Value at a point $c: \varphi(c)$;

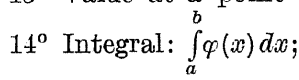

$15^{\circ}$ Convolution: $\varphi(x) * \psi(x)$;

$16^{\circ}$ Fourier transform.

It is essential to distinguish between the operations $5^{\circ}$ and $11^{\circ} ; 5^{\circ}$ is interpreted as an operation on a singie function $\varphi(x)$, the coefficient $\omega(x)$ being fixed; $11^{\circ}$ is interpreted as an operation on a pair of functions. $5^{\circ}$ is a regular operation, $11^{\circ}$ is an irregular operation. Similar remark concerns $6^{\circ}$ and $12^{\circ}$ as well as $9^{\circ}$ and $15^{\circ}$.

We are going to give a general method of extension of irregular operations. This method can also be applied to regular operations and yields then the same result.

For every distribution $f(x)$ we select from among its fundamental sequences some particular sequences; those will be called regular sequences. 
Then, given any operation $R(p, \psi, \ldots)$, regular or irregular, we shall say that it is feasible on distributions $f, g, \ldots$, if for any regular sequences $\varphi_{n}, \psi_{n}, \ldots$ which belong to $f, g, \ldots$ respectively the sequence $R\left(\gamma_{n}, \psi_{n}, \ldots\right)$ is fundamental. In that case we put, as in $\$ 3$,

$$
R(f, g, \ldots)=\left[R\left(p_{n}, \psi_{n}, \ldots\right)\right] .
$$

In order to ensure the uniqueness of the resulti it suffices to suppose the following properties of regular sequences:

(I) Every subsequence of a regular sequence is a regular sequence;

(II) If $p_{n}$ and $\psi_{n}$ are regular sequences for the same distributions, then the interlacing sequence $p_{1}, w_{1}, p_{2}, w_{2}, \ldots$ is also a regular sequence for that distribution.

Since regular sequences are fundamental sequences, the oxtension by means of regular sequences coincides, in the asso of regular operations, with the extension considered in $\S 3$.

5. Operations preserving continuity. $\Lambda$ s starting points in the preceding paragraphs we took the operations which are feasible on smooth functions. But in particular cases it is used to formulate definitions directly for a larger ciass of functions, e. g. for continuous functions. But continuous functions can be considered as distributions so that we obtain by the preceding method alternative definitions of operations on continuous functions. There arises the question of compatibility of those definitions with direct ones.

Suppose that an operation $R(p, \psi, \ldots)$ defined for all smooth functions is also defined for some (or all) continuous functions and satisfies the following condition:

(C) If sequences of smooth functions $\varphi_{n}, \psi_{n}, \ldots$ converge almost uniformly to continuous functions $f, g, \ldots$ respectively, then the sequence $R\left(\varphi_{n}, \psi_{n}, \ldots\right)$ converges almost uniformly to $R(f, g, \ldots)$.

Evidently the operations $1^{\circ}-7^{\circ}, 9^{\circ}-13^{\circ}$ and also $14^{\circ}$, provided the interval of integration is compact, satisfy condition $(\mathrm{O})$.

In order to ensure the compatibility in case of operations with property (C) it suffices to impose on regular sequences the following condition:

(III) If a distribution is, in an interval, a continuous function, then each of its. regular sequences converges almost uniformly in that interval.

6. Operations preserving integrability. Wo shall say that a sequence of smooth functions $\varphi_{n}$ is $L$-convergent, if it converges almost everywhere and, moreover, if in every compact interval $[a, b]$ the integral

$$
\int_{a}^{x} \varphi_{n}(t) d t
$$

converges uniformly. Then the limit of $p_{n}$ is of course a locally integrable function $f$ and the limit of $(1)$ is $\int_{a}^{x} f(t) d t$. In case of dimension 0 , every convergent sequence (of numbers) is said to be $L$-convergent.

Suppose that an operation $R(\varphi, \psi, \ldots)$, defined for all smooth functions, is also defined for some (or all) locally integrable functions and satisfies the following condition:

(L) If sequences $\varphi_{n}, \psi_{n}, \ldots$ are L-convergent to locally integrable functions $f, g, \ldots$ respectively, then the sequence $R\left(\varphi_{n}, \psi_{n}, \ldots\right)$ is L-convergent to $R(f, g, \ldots)$.

It is easy to verify that the operations $1^{\circ}-7^{\circ}, 9^{\circ}, 10^{\circ}$ and also $14^{\circ}$, provided the interval of integration is compact, satisfy condition ( $\mathrm{L}$ ).

In order to ensure the compatibility of the distributional extension of operations satisfying ( $\mathrm{L}$ ) with their direct definitions for locally integrable functions it suffices to impose on regular sequences the following property:

(IV) If a distribution is, in an interval, a locally integrable function, then each of its regular sequences is L-convergent in that interval.

The operations $8^{\circ}, 15^{\circ}$ and $16^{\circ}$ preserve neither continuity nor integrability. Nevertheless those operations are also defined directly for some subclasses of continuous or integrable functions. The problem of compatibility is then more complicated and we do not intend to discuss it in this paper.

7. A set of regular sequences. It is possible to define regular sequences an a lot of different ways. For compatibility sake these sequences should satisfy some complementary conditions; anyhow it is natural to require conditions (I)-(IV). In the sequel, we are going to give a definition of regular sequences which seems to be adequate. First we define regular sequences for the Dirac delta function, or shortly, regular $\delta$-sequences.

Under such a sequence we understand every sequence of smooth functions $\delta_{n}(x)=0$ outside $\left(-\frac{1}{2} \varepsilon_{n} e, \frac{1}{2} \varepsilon_{n} e\right)$, where $\varepsilon_{n}$ is a sequence of positive numbers convergent to $0, e=(1, \ldots, 1)$, and moreover,

$$
\int_{-\infty}^{\infty} \delta_{n}(x) d x=1, \quad\left|\delta_{n}(x)\right|<\frac{M}{\varepsilon_{q}} .
$$

As an example we can take

$$
\delta_{n}(x)=\frac{1}{\varepsilon_{n}^{q}} \Delta\left(\frac{x}{\varepsilon_{n}}\right),
$$



where $\Delta(x)$ is any smooth function vanishing outside the interval $(-e, e)$
and such that

$$
\int_{-\infty}^{\infty} A(x) d x=1
$$

Evidently, every regular: $\delta$-sequence is a fundamontal sequence of delta function. The conditions (I) and (II) are satisfied, and, strictly speaking, so are (III) and (IV), for delta function is neither a continuous
nor an integrable function.

Under a regular sequence of a given distribution we shall understand overy sequenco

$$
\varphi_{n}=f * \delta_{n}=\int_{-\infty}^{\infty} f(x-t) \delta_{n}(t) d t .
$$

One can verify that these sequences are fundamental sequences and satisfy conditions (I), (II), (III). We are going to show that also the con-
dition (IV) is satisfied.

Suppose that $f$ is a locally integrable function. Then, for almost every point $x_{0}$, we have

$$
\lim _{\varepsilon \rightarrow 0+} \frac{1}{\varepsilon^{\prime l}} \int_{I_{s}} f\left(x_{0}-t\right) d t=f\left(x_{0}\right)
$$

where $I=\left(-\frac{1}{2} \varepsilon e, \frac{1}{2} \varepsilon e\right)$. We shall show that for those points $x_{0}$ we have

$$
\lim _{n \rightarrow \infty} p_{n}\left(x_{0}\right)=f\left(x_{0}\right) \text {. }
$$
There is no loss of generality when assuming that $f\left(x_{0}\right)=0$. Then we
have

Moreover

$$
\left|\varphi_{n}\left(x_{0}\right)\right| \leqslant \int_{-\infty}^{\infty}\left|f\left(x_{0}-t\right) \delta_{n}(t)\right| d t \leqslant \frac{M}{\varepsilon_{n}^{\prime}} \int_{x_{c_{n}}}\left|f\left(x_{0}-t\right)\right| d t \rightarrow 0 .
$$

$$
\begin{aligned}
\int_{a}^{x} f_{n}(b) d t-\int_{a}^{x} f(t) d t & =\int_{a}^{x} d t \int_{-\infty}^{\infty}(f(t-\tau)-f(t)) \delta_{n}(\tau) d \tau \\
& =\int_{I_{\varepsilon}}(F(x-\tau)-F(x)) \delta_{n}(\tau) d \tau,
\end{aligned}
$$

where $F(x)=\int_{a}^{x} f(t) d t$. In view of continuity of $H^{\prime}(x)$, this implies that

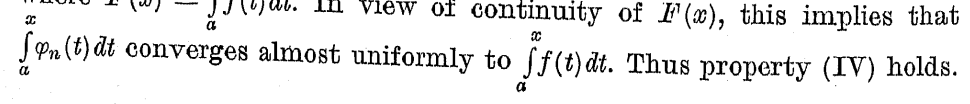

In order to ensure other properties of regular sequences, it may be useful to introduce further restrictions on $\delta_{n}(x)$, e. g.

$$
\left|\delta_{n}^{(k)}(x)\right|<\frac{M_{k}}{\varepsilon^{q+|k|}} \quad\left(|k|=\varkappa_{1}+\ldots+\varkappa_{q}\right) .
$$

\section{References}

[1] S. Łojasiewicz, Sur la valeur et la limite d’une distribution dans un point, Studia Math. 16 (1957), p. $1-36$.

[2] J. Mikusiński, Sur la méthode de généralisation de M. Laurent Schwartz et sur la convergence faible, Fund, Math. 35 (1948), p. $235-239$.

[3] J. Mikusiński and R. Sikorski, The elementary theory of distributions (I), Rozprawy Matematyczne 12 (1957).

[4] - The elementary theory of distributions (II), to appear in Rozprawy Matematyczne.

[5] L. Schwartz, Théorie des distributions I et II, Paris 1950 et 1951.

[6] G. Temple, Theories and applications of generalized functions, Journ. of the London Math. Soc. 28 (1953), p. 134-148.

Reçu par la Rédaction le 19. \%. 1960 